\title{
Relating multichannel scattering and production amplitudes in a microscopic OZI-based model
}

\author{
Eef van Beveren ${ }^{\text {a }}$, George Rupp ${ }^{\text {b,* }}$ \\ ${ }^{a}$ Centro de Física Teórica, Departamento de Física, Universidade de Coimbra, P-3004-516 Coimbra, Portugal \\ ${ }^{\mathrm{b}}$ Centro de Física das Interacções Fundamentais, Instituto Superior Técnico, Universidade Técnica de Lisboa, \\ Edifício Ciência, P-1049-001 Lisboa, Portugal
}

Received 27 June 2007; accepted 19 November 2007

Available online 15 December 2007

\begin{abstract}
Relations between scattering and production amplitudes are studied in a microscopic multichannel model for meson-meson scattering, with coupling to confined quark-antiquark channels. Overlapping resonances and a proper threshold behaviour are treated exactly in the model. Under the spectator assumption, it is found that the two-particle production amplitude shares a common denominator with the elastic scattering amplitude, besides a numerator consisting of a linear combination of all elastic and some inelastic matrix elements. The coefficients in these linear combinations are shown to be generally complex. Finally, the standard operator expressions relating production and scattering amplitudes, viz. $A=T / V$ and $\mathfrak{I} m(A)=T^{*} A$, are fulfilled, while in the small-coupling limit the usual isobar model is recovered.
\end{abstract}

(C) 2007 Elsevier Inc. All rights reserved.

PACS: $11.80 . \mathrm{Gw} ; 11.55 . \mathrm{Ds} ; 13.75 . \mathrm{Lb} ; 12.39 . \mathrm{Pn}$

Keywords: Relating scattering and production amplitudes; Multichannel meson-meson scattering; Analytically solvable microscopic coupled-channel model; OZI-allowed processes; Generalised unitarity relations; Universal $S$-matrix poles

\footnotetext{
${ }^{*}$ Corresponding author. Fax: +351 218419143.

E-mail addresses: eef@teor.fis.uc.pt (E. van Beveren), george@ist.utl.pt (G. Rupp).
} 


\section{Introduction}

In a very recent article [1] we have shown that several hadronic three-body decays of $J / \psi, D$ and $D_{s}$ mesons can be well described, up to moderately high energies, in a model for production processes derived from the so-called Resonance-Spectrum Expansion (RSE) [2]. The RSE formalism amounts to an effective description of non-exotic meson-meson scattering, based upon quark-antiquark pair creation and annihilation allowing transitions between an infinity of confined $q \bar{q}$ states and the meson-meson continuum. An essential feature of the RSE is that it gives rise to closed-form expressions for the $S$-matrix and even the fully off-shell $T$-matrix. Hence, exact analyticity and unitarity properties, as well as a correct (sub)threshold behaviour, are manifestly satisfied. Moreover, the resulting meson-meson production amplitude can be derived exactly, too, by summing the corresponding two-body Born series, the only assumption being that the third particle acts as a mere spectator [1].

In the present paper, we shall further develop the formalism introduced in Ref. [1], so as to cover the most general multichannel case in mesonic three-body decays. Clearly, at higher energies competing inelastic two-meson channels require that the production amplitude be described by a vector and not a scalar function. The underlying two-body scattering $T$-matrix is then a true matrix. Furthermore, also the quark-antiquark sector needs an extension, as there can be mixing of different $q \bar{q}$ channels that couple to the same mesonmeson channels. This is the case in, e.g., the production of $I=0 \pi \pi$ and $K \bar{K}$ pairs, which both couple to the $n \bar{n}(=(u \bar{u}+d \bar{d}) / \sqrt{2})$ and $s \bar{s}$ channels, giving rise to the isoscalar scalar resonances $f_{0}(600)$ (alias $\sigma$ ) and $f_{0}(980)$. Finally, having a general and exact - within the model assumptions - expression for production amplitudes at hand, we may carry out a detailed comparison with the ansatzes employed in other approaches, focusing on common features as well as clear differences.

Under the spectator approximation, we assume that a pair of mesons is created out of one $q \bar{q}$ pair emerging from the original decay, accompanied by a non-interacting spectator meson. In this process, we only consider OZI-allowed [3] strong transitions to pairs of mesons. The intensities of transitions between the initial $q \bar{q}$ pair (its quantum numbers, including flavour, are abbreviated by $\alpha$ ) and the various meson-meson pairs $i$ allowed by quantum numbers are given by coupling constants $g_{\alpha i}$ determined via the recoupling scheme of Ref. [4]. In Section 3, we derive the matrix elements $t(i \rightarrow v, E)$ of the scattering amplitude at total CM energy $E=\sqrt{s}$, for transitions between the meson-meson channels $i$ and $v$. In the same section, we establish a relation between the common denominator $D(E)$ of all matrix elements $t$ (anything $\rightarrow$ anything, $E$ ) and the numerators of diagonal matrix elements of $t(E)$, the latter representing elastic scattering. The production amplitude $a(\alpha \rightarrow i, E)$, which is related to the probability of producing a meson-meson pair $i$, assuming that a $q \bar{q}$ pair emerges in the initial - here not described - stages of the decay process, is determined in Section 4. Note that the process giving rise to the initial $q \bar{q}$ pair plus the spectator meson can be either weak or strong yet OZI-suppressed (see Ref. [1] for some examples).

The central result of the present paper is a relation between the production and scattering amplitudes which can be formulated as

$$
a(\alpha \rightarrow i, E) \propto \frac{g_{\alpha i}}{D(E)}+i \lambda^{2} \sum_{v}\left\{g_{\alpha i} x_{v}(E) t(v \rightarrow v, E)-g_{\alpha, v} x_{i}(E) t(i \rightarrow v, E)\right\}
$$


where the $x_{i}$ stand for momentum distributions that will be specified in Section 4 (Eq. (19)).

We thus obtain the result that the production amplitude is in the first place given by the common denominator of the scattering amplitudes. This implies that, within the RSE formalism, resonance poles are identical for production and scattering, at least in the spectator approximation. Secondly, we find that the remainder of the production amplitude to the $i$ th two-meson channel is proportional to the sum of the differences between all possible elastic scattering amplitudes $t(v \rightarrow v, E)$ and the inelastic amplitudes for the $i$ th channel, $t(i \rightarrow v, E)$. This does not spoil our conclusion about the resonance poles, since all $T$-matrix elements share the common denominator. Note, however, that the contribution of the term $v=i$ vanishes in the expression between braces on the r.h.s. of Eq. (1). Consequently, the amplitude $a(\alpha \rightarrow i, E)$ for the production of a two-meson pair $i$ does not carry any dependence on the amplitude for elastic scattering $i \rightarrow i$. In those cases where the coupling to different meson-meson channels vanishes or can be neglected, implying a $1 \times 1 T$-matrix, the resulting production amplitude is solely determined by the common denominator $D(E)[1]$.

Some words are due about the $K$-matrix formalism. In general, and so also here, the $T$ matrix can be written as $T=K /(1-i K)$, where $K$ is a real symmetric matrix. So, at first sight, it seems that the common denominator of all $T$-matrix elements is given by $1-i K$. However, this is not the case. First of all, $K$ is a matrix and not just a real function. But even in the $1 \times 1$ case, where $K$ can be represented by a real function, it has a denominator itself, the zeros of which are the $K$-matrix poles, located at the real energies where some eigenphase shift passes through $90^{\circ}$. The common denominator above is then the sum of the denominator of $K$ plus $-i$ times the numerator of $K$. In general, when $K$ is a matrix, this relation involves the determinant of $K$. Hence, comparing $K$-matrix poles, lying on the real axis in the complex energy plane, and resonance poles, which are usually in the second Riemann sheet with respect to the nearest "open" threshold, is far from trivial. Moreover, for some resonances, like the $\sigma$ and the $\kappa\left(K_{0}^{*}(800)\right.$ [5]), the respective $K$-matrix poles, corresponding to an elastic phase shift $\delta=90^{\circ}$, lie about $350-600 \mathrm{MeV}$ higher in energy that the real parts of the respective $S$-matrix poles, while mixing with other resonances $\left(f_{0}(980)\right.$ and $\left.K_{0}^{*}(1430)\right)$ further complicates the picture. So rather than making ad hoc assumptions about poles of the production amplitude, we shall straightforwardly derive the latter, and then see what its properties are.

A final remark here concerns Watson's [6] theorem for production. This theorem implicitly relies on having a potential which is energy independent or only weakly energy dependent. However, this is not the case here, because the energy dependence of our effective meson-meson potential is far stronger than that of the scattering $T$-matrix. As a consequence, the energy dependence of the production experiment does not resemble at all the one of the $T$-matrix, and all exercises imposing the Watson "theorem" or theorems derived from it are inappropriate here. This issue is analysed in more detail in Ref. [7].

\section{The Resonance-Spectrum Expansion (RSE)}

Scattering from a weakly coupled resonant source has been studied in a variety of different approaches. For such systems it is observed that resonances occur at energies that are close to the unperturbed spectrum of the resonant source. Widths and mass shifts can 
be determined by perturbative methods, and expressed in terms of pole positions of the resonances in the complex energy plane.

Intuitively, however, perturbative methods do not offer the correct strategy for strong interactions. Since in the present paper we are interested in obtaining exact relations between scattering and production amplitudes, which are moreover based on a microscopic description in terms of quarks, we rather fall back upon an approximate yet exactly solvable theory or model. Such a manifestly unitary and analytic framework is provided by the RSE.

The RSE aims at describing the scattering of meson pairs in non-exotic channels, thereby assuming that in the interaction region a meson pair may temporarily transform into a quark-antiquark pair through $q \bar{q}$ annihilation and subsequent creation. The transitions of the system, from meson-meson pairs to $q \bar{q}$ pairs and vice versa, are described by an off-diagonal potential $V_{t}$ in the RSE, linking these two sectors to each other. It has a maximum at an interquark distance $r_{0}$ which depends on the average effective quark mass and runs from slightly less than $0.2 \mathrm{fm}$ for $b \bar{b}$ to about $0.6 \mathrm{fm}$ for light quarks. Furthermore, we assume that this mechanism gives rise to the dominant meson-meson interaction in non-exotic channels. Here, we limit ourselves to the case where $V_{t}$ is considered the only interaction.

The intermediate $q \bar{q}$ states are supposed to have an unperturbed confinement spectrum depending on the quantum numbers of the system. Its energy eigenvalues are all contained in the two-meson scattering matrix, which, consequently, develops corresponding CDD resonance poles.

An additional nice feature of the RSE, which will turn out to be crucial for the construction of the production amplitude, is the possibility to obtain the closed-form scattering $T$-matrix both in configuration and in momentum space. In the former representation, coupled-channel Schrödinger equations with the proper boundary conditions directly lead to the full solution, while in the latter picture individual Born terms can be explicitly calculated and then exactly summed up owing to the general separable nature of the effective meson-meson potential, with no need to solve the Lippmann-Schwinger [8] integral equations. This allows to verify the correctness of the momentum space approach in the scattering case, which is the only method at our disposal to describe production. As we shall see below, a similar Born series can then be written down and summed up.

\section{Scattering}

The building blocks of the RSE meson-meson scattering amplitude are the effective meson-meson potentials and the free two-meson propagators, which are graphically represented in Fig. 1. It is the philosophy of the RSE that confinement and decay can be sep-

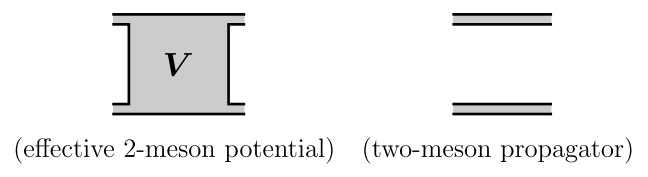

Fig. 1. Graphical representation of the building blocks of the RSE two-meson scattering amplitude. The solid lines represent valence quarks and antiquarks as in usual Feynman diagrams. In contrast, the grey areas stand for all possible confining interactions, like gluon exchange, sea-quark loops and their higher orders. The effective meson-meson interaction is represented by $V$. Furthermore, although the mesons in the two-meson propagators are considered point-like in the RSE, for clarity they are here represented by double quark lines connected by confining interactions. 
arated. Hence, in the interaction region, a two-meson system can appear as a permanently confined system consisting of a valence quark and a valence antiquark. Possible intermediate crypto-exotic multiquark states are not considered in the RSE.

The dynamics of the intermediate $q \bar{q}$ states is described by a permanently confining Hamiltonian $H_{c}$, which has a complete set of eigenstates at eigenvalues representing the confinement spectrum. The other part of the strong interactions, generating transitions between a two-meson system and a $q \bar{q}$ state, is given by a transition potential $V_{t}$. Consequently, the effective meson-meson interaction in Fig. 1 is described by the operator

$$
V=V_{t}^{T}\left[E-H_{c}\right]^{-1} V_{t},
$$

where $E$ is the total invariant mass of the coupled-channel system. These interactions and the free two-meson propagators, both depicted in Fig. 1, can then be used in an ordinary Lippmann-Schwinger [8] approach to scattering. Nevertheless, in constructing the Born series, quark-loop contributions to all orders are automatically accounted for, as becomes clear from Fig. 2.

The RSE scattering amplitude depicted in this figure has the Born term

$$
V(i \rightarrow j)=\left\langle i, \vec{p}_{i}\left|V_{t}^{T}\left[E(p)-H_{c}\right]^{-1} V_{t}\right| j, \vec{p}_{j}\right\rangle .
$$

In Ref. [2] it was shown how, under the RSE assumptions, the integrations can be done analytically. For the present discussion, it is only necessary to mention the generic form of the RSE expression, given by

$$
V(i \rightarrow j)=\frac{\lambda^{2}}{4 \pi^{2}} \sum_{\ell=0}^{\infty}(2 \ell+1) P_{\ell}\left(\hat{p}_{i} \cdot \hat{p}_{j}\right) j_{\ell}\left(p_{i} r_{0}\right) j_{\ell}\left(p_{j} r_{0}\right) Z_{i j}^{(\ell)}(E)
$$

The overall coupling $\lambda$ and the interaction radius $r_{0}$ represent the total probability of quark-pair creation/annihilation and the average interquark distance at which such processes take place, respectively; $j_{\ell}$ stands for the spherical Bessel function for the relative meson-meson angular momentum $\ell ; \vec{p}_{i}$ and $\vec{p}_{j}$ are the relative linear momenta in the two-meson channels $i$ and $j$, respectively. The matrix $Z$ is a real and symmetric function of the total invariant mass of the system.

The intermediate $q \bar{q}$ systems may have different orbital angular momenta, for the same quantum numbers. For example, a meson-meson system with $J^{P C}=1^{--}$couples to $q \bar{q}$ systems in either an $S$ or a $D$ wave. On the other hand, isosinglet mesons can be mixtures of different quark flavours, usually $n \bar{n}$ and $s \bar{s}$. In such cases, more than one type of $q \bar{q}$ states are involved in the quark loops of the process depicted in Fig. 2. However, since the recurrencies of the permanently confined $q \bar{q}$ systems are automatically included by the definition of the Born term (4), the number of $q \bar{q}$ channels that couple to a specific set of two-meson quantum numbers is limited, usually to one or two. Nevertheless, the number of two-meson scattering channels is in principle not restricted.

For any number of coupled confinement and scattering channels, the general structure of the amplitude reads $\left(E=E\left(p_{i}\right)=E\left(p_{j}\right)\right)$

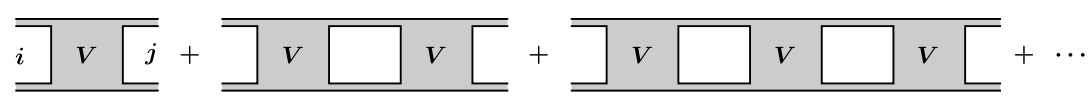

Fig. 2. Graphical representation of the RSE scattering amplitude. 


$$
\begin{aligned}
t(i & \rightarrow j)=\left\langle i, \vec{p}_{i}|t| j, \vec{p}_{j}\right\rangle=\left\langle i, \vec{p}_{i}|(V+V G V+V G V G V+\ldots)| j, \vec{p}_{j}\right\rangle \\
& =\frac{\lambda^{2}}{4 \pi^{2}} \sum_{\ell=0}^{\infty}(2 \ell+1) P_{\ell}\left(\hat{p}_{i} \cdot \hat{p}_{j}\right) j_{\ell}\left(p_{i} r_{0}\right) j_{\ell}\left(p_{j} r_{0}\right) \frac{\mathcal{A}_{i j}^{(\ell)}(E)}{\mathcal{D}^{(\ell)}(E)}
\end{aligned}
$$

where $\mathcal{A}$ and $\mathcal{D}$ are functions of the total invariant mass $E$ satisfying the unitarity condition

$$
\mathfrak{I} m\left(\mathcal{D}^{(\ell)} \mathcal{A}_{i j}^{(\ell)^{*}}\right)=2 \lambda^{2} \sum_{v} \mu_{v} p_{v} j_{\ell}^{2}\left(p_{v} r_{0}\right) \mathcal{A}_{i v}^{(\ell)} \mathcal{A}_{j v}^{(\ell)^{*}}
$$

The denominator $\mathcal{D}$ contains the full pole structure of the coupled two-meson states. In order to be a bit more specific, let us consider the scattering of charmed mesons, i.e., $D \bar{D}, D^{*} \bar{D}, D^{*} \bar{D}^{*}, D_{s} \bar{D}_{s}, D_{s}^{*} \bar{D}_{s}$ and $D_{s}^{*} \bar{D}_{s}^{*}$, all coupled to $c \bar{c}$. For such a process, $\mathcal{D}$ has in the RSE the form

$$
\mathcal{D}^{(\ell)}(E)=1+2 i \lambda^{2} \sum_{v} g_{v}^{2}\left\{\sum_{n=0}^{\infty} \frac{\left|F_{c \bar{c}}^{(n)}\left(r_{0}\right)\right|^{2}}{E-E_{n}}\right\} \mu_{v} p_{v} j_{\ell}\left(p_{v} r_{0}\right) h_{\ell}^{(1)}\left(p_{v} r_{0}\right),
$$

where the outer sum runs over all two-meson channels, and the inner sum over all recurrencies $n$ for the operator $H_{c}$ describing confinement in the $c \bar{c}$ system. $F_{c \bar{c}}^{(n)}$ and $E_{n}$ represent the eigenstate and eigenvalue of the $n$th recurrency of the of $H_{c}$ spectrum, respectively. Furthermore, the $g_{v}$ stand for the relative couplings of each of the two-meson systems to $c \bar{c}$, while $h_{\ell}^{(1)}$ is a spherical Hankel function of the first kind.

The denominator $\mathcal{D}(E)$ vanishes for $E$ near $E_{n}$ and small overall coupling $\lambda$. In this case, the scattering cross sections in all channels display narrow spikes for values of $E$ in the vicinity of $E_{n}(n=0,1,2, \ldots)$. Hence, for small $\lambda$, the theoretical cross sections reproduce - up to small shifts - the hypothetical $c \bar{c}$ confinement spectrum.

However, for larger values of $\lambda$ the zeros in $\mathcal{D}$ are no longer near the eigenvalues of $H_{c}$, but move deeper into the complex $E$ plane, farther away from the real axis and with appreciable shifts for the real parts as well. Then, the resonance spectrum does no longer reproduce the spectrum of $H_{c}$ : resonances start overlapping and even the number of zeros in $\mathcal{D}$ that lie close enough to the real energy axis to be observed experimentally may change. We believe this describes quite accurately the true situation in hadron spectroscopy.

Below the lowest threshold, poles, i.e., zeros in $\mathcal{D}$ (Eq. 7), come out on the real axis, because the expression $i j_{\ell} h_{\ell}^{(1)}$ turns real. In that case, expression (5) describes bound $c \bar{c}$ states, such as $\eta_{c}, J / \psi, \chi_{c}(1 P)$ and $\psi(2 S)$, yet with an admixture of two-meson components. The energy eigenvalues of these "dressed" states then depend on the value of $\lambda$. It has been observed $[9,10]$ that charmonium mass shifts with respect to the pure confinement spectrum can be surprisingly large in the RSE, as well as in other approaches [11].

In the present work, we intend to derive relations among $\mathcal{A}_{i j}^{(\ell)}, \mathcal{D}^{(\ell)}$ and $Z_{i j}^{(\ell)}$. In principle, this could be achieved by just performing the calculus outlined in Ref. [2]. However, here we shall allow more general expressions for the $Z$ matrix in the Born term (4). Hence, apart from the unitarity condition (6), we must construct a second relation. For that purpose, we write the identity 


$$
\begin{aligned}
0= & \left\langle i, \vec{p}_{i}|(T-V-T G V)| j, \vec{p}_{j}\right\rangle \\
= & \frac{\lambda^{2}}{4 \pi^{2}} \sum_{\ell=0}^{\infty}(2 \ell+1) P_{\ell}\left(\hat{p}_{i} \cdot \hat{p}_{j}\right) j_{\ell}\left(p_{i} r_{0}\right) j_{\ell}\left(p_{j} r_{0}\right) \\
& \times\left\{\frac{\mathcal{A}_{i j}^{(\ell)}}{\mathcal{D}^{(\ell)}}-Z_{i j}^{(\ell)}+2 i \lambda^{2} \sum_{v} \mu_{v} p_{v} j_{\ell}\left(p_{v} r_{0}\right) h_{\ell}^{(1)}\left(p_{v} r_{0}\right) \frac{\mathcal{A}_{i v}^{(\ell)}}{\mathcal{D}^{(\ell)}} Z_{v j}^{(\ell)}\right\},
\end{aligned}
$$

which yields the relation

$$
\mathcal{D}^{(\ell)} Z_{i j}^{(\ell)}=\mathcal{A}_{i j}^{(\ell)}+2 i \lambda^{2} \sum_{v} \mu_{v} p_{v} j_{\ell}\left(p_{v} r_{0}\right) h_{\ell}^{(1)}\left(p_{v} r_{0}\right) \mathcal{A}_{i v}^{(\ell)} Z_{v j}^{(\ell)}
$$

Furthermore, if we assume

$$
\mathcal{A}_{i j}^{(\ell)}=\mathcal{A}_{i j}^{(\ell)(0)}+\lambda^{2} \mathcal{A}_{i j}^{(\ell)(1)}+\lambda^{4} \mathcal{A}_{i j}^{(\ell)(2)}+\cdots,
$$

then we obtain the following solution to relations (6) and (9):

(1) The denominator $\mathcal{D}$ can be fully expressed in terms of the numerators $\mathcal{A}$, according to

$$
\mathcal{D}^{(\ell)}=1+2 i \lambda^{2} \sum_{v} \mu_{v} p_{v} j_{\ell}\left(p_{v} r_{0}\right) h_{\ell}^{(1)}\left(p_{v} r_{0}\right) \mathcal{A}_{v v}^{(\ell)} .
$$

(2) The zeroth-order term of (10) is evidently given by the Born term (4):

$$
\mathcal{A}_{i j}^{(\ell)(0)}=Z_{i j}^{(\ell)} .
$$

(3) For the higher-order terms of the expansion (10) we obtain the recursion relation

$$
\mathcal{A}_{i j}^{(\ell)(n+1)}=2 i \sum_{v} \mu_{v} p_{v} j_{\ell}\left(p_{v} r_{0}\right) h_{\ell}^{(1)}\left(p_{v} r_{0}\right)\left\{\mathcal{A}_{v v}^{(\ell)(n)} Z_{i j}^{(\ell)}-\mathcal{A}_{i v}^{(\ell)(n)} Z_{v j}^{(\ell)}\right\} .
$$

From Eq. (5) we then get a partial-wave scattering amplitude of the form

$$
t_{\ell}(i \rightarrow j)=2 \lambda^{2} j_{\ell}\left(p_{i} r_{0}\right) j_{\ell}\left(p_{j} r_{0}\right) \frac{\mathcal{A}_{i j}^{(\ell)}(E)}{\mathcal{D}^{(\ell)}(E)} .
$$

For a full definition of this amplitude, satisfying the unitarity conditions for scattering, see Eq. (A.1).

\section{Production}

Various opinions exist on how to analyse the final-state interactions of pairs of hadrons emerging from a decay process [12-15]. In particular, the production of pion pairs has been studied from many different angles. Several resonances have been discovered and established in this channel. However, there still are many open questions, of which the most intriguing one probably is the formation of the $f_{0}(980)$ resonance [16-26]. As such, this resonance seems to be one of the key issues for understanding strong interactions. It lies close to the $K \bar{K}$ threshold, couples relatively weakly to pions, comes on top of a much 
broader structure, namely the $f_{0}(600)$, and is furthermore not very distant from a broad resonance around $1.35 \mathrm{GeV}$, viz. the $f_{0}(1370)$ [15].

It is our understanding that mesonic resonances, like the $f_{0}(600)$ and the $f_{0}(980)$, form an integral part of the whole meson family. Therefore, we have developed a model for all $q \bar{q}$ phenomena, including those involving charm and bottom. Here, we wish to develop a new tool for data analysis, which is an amplitude for the description of final-state interactions in two-meson subsystems emerging in decay processes involving other particles. This production amplitude is based on the two-meson scattering amplitude given in Eq. (5).

For the description of the final-state interactions of meson pairs in production processes, it is common practice to make the spectator assumption, according to which the other emerging hadrons do not interact strongly with the pair. Evidently, this is an approximation, which is justified by the observation that in most production processes resonances involving the third (or fourth, ...) hadron are much higher in mass than the energies considered for the pair. Here, we moreover assume that the meson pair is generated from an initially produced $q \bar{q}$ pair. Our amplitude for the production of a meson pair, including all higher-order contributions from final-state interactions, is depicted in Fig. 3. Also using expression (5) for the scattering amplitude, we are led to define for the production amplitude

$$
\begin{aligned}
a(\alpha \rightarrow & i)=\left\langle i, \vec{p}_{i}\left|(1+T G) V_{t}\right|(q \bar{q})_{\alpha}, E\right\rangle \\
= & \left\langle i, \vec{p}_{i}\left|V_{t}\right|(q \bar{q})_{\alpha}, E\right\rangle+\sum_{v} \int d^{3} k_{v}\left\langle i, \vec{p}_{i}|T| v, \vec{k}_{v}\right\rangle G\left(\vec{k}_{v}\right)\left\langle v, \vec{k}_{v}\left|V_{t}\right|(q \bar{q})_{\alpha}, E\right\rangle \\
= & \frac{\lambda}{\sqrt{\pi}} \sum_{\ell, m}(-i)^{\ell} j_{\ell}\left(p_{i} r_{0}\right) Y_{m}^{(\ell)}\left(\hat{p}_{i}\right) Q_{\ell_{q \bar{q}}}^{(\alpha)}(E) \\
& \times\left\{g_{\alpha i}-2 i \lambda^{2} \sum_{v} \mu_{v} p_{v} j_{\ell}\left(p_{v} r_{0}\right) h_{\ell}^{(1)}\left(p_{v} r_{0}\right) g_{\alpha v} \frac{\mathcal{A}_{i v}^{(\ell)}(E)}{\mathcal{D}^{(\ell)}(E)}\right\} .
\end{aligned}
$$

Here, $Q_{\ell_{q \bar{q}}}^{(\alpha)}$ represents the overlap with the initial $q \bar{q}$ distribution, having quantum numbers $\alpha$ and relative interquark angular momentum $\ell_{q \bar{q}}$. Notice that the latter quantum number is related - though unequal - to the relative two-meson angular momentum $\ell$, because of total-angular-momentum and parity conservation. Below, we shall discuss the properties of production amplitude (15) for pairs of interacting mesons.

\section{1. $P_{i}=\sum_{v} c_{v} T_{v i}$ ?}

The result (15) agrees to some extent with the expression proposed in Refs. [27,28]. Like here, the authors of Ref. [28] based their ansatz on the OZI rule [3] and the spectator picture, so as to find that the production amplitude can be written as a linear combination of

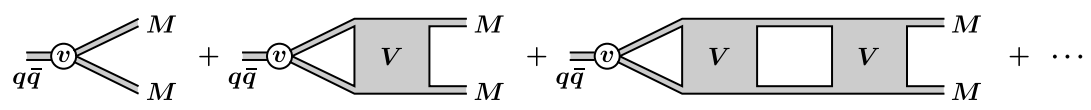

Fig. 3. Graphical representation of the RSE production amplitude. The transition $q \bar{q} \rightarrow M M$, denoted by $V_{t}$ in the text, is here represented by $v$; the resulting effective $M M$ interaction is denoted by $V$. 
the elastic $t_{\ell}(i \rightarrow i)$ and inelastic $t_{\ell}(i \rightarrow v \neq i)$ scattering amplitudes, with coefficients that do not carry any singularities, but are rather supposed to depend smoothly on the total CM energy of the system.

Indeed, if we carry out the substitution (14), we find for our production amplitude the expression

$$
\begin{aligned}
a(\alpha \rightarrow i)= & \frac{\lambda}{\sqrt{\pi}} \sum_{\ell, m}(-i)^{\ell} Y_{m}^{(\ell)}\left(\hat{p}_{i}\right) Q_{\ell_{\bar{q}}}^{(\alpha)}(E) \\
& \times\left\{g_{\alpha i j} j_{\ell}\left(p_{i} r_{0}\right)-i \sum_{v} \mu_{v} p_{v} h_{\ell}^{(1)}\left(p_{v} r_{0}\right) g_{\alpha v} t_{\ell}(i \rightarrow v)\right\},
\end{aligned}
$$

which contains a linear combination of elements of the scattering amplitude, with coefficients smooth in $E$.

However, Ref. [28] concluded from the relation

$$
\mathfrak{I} m(A)=T^{*} A
$$

that the production amplitude must be given by a real linear combination of the elements of the transition matrix. A similar conclusion, based on a $K$-matrix parametrisation, can be found in Ref. [29]. In contrast, we arrive at a different conclusion, namely that, as the Hankel function of the first kind is a complex function for real arguments, the coefficients must be complex, in agreement with experimental analyses [13,30,31] as well as with the theoretical work of the Ishidas [32,33].

Relation (17), which can be also found in Ref. [34] basically stems from the operator relations $A V=(1+T G) V=V+T G V=T$, the symmetry of $T$, the realness of $V$ and the unitarity of $1+2 i T$, which gives $\mathfrak{I} m(A) V=\mathfrak{I} m(A V)=\mathfrak{I} m(T)=T^{*} T=T^{*} A V$. This leads, for non-singular potentials $V$, to relation (17). In Appendix A, we show that notwithstanding the complex coefficients in Eq. (16), relation (17) is satisfied for the scattering and production amplitudes of Eqs. (5) and (15), respectively. Consequently, relation (17) does not impose a realness condition on the coefficients in Eq. (16).

\subsection{The lowest-order term}

Besides the sum over transition matrix elements, our production amplitude (16) also contains an extra term $\propto g_{\alpha i} j_{\ell}\left(p_{i} r_{0}\right)$. Such a term was not considered in Refs. [27-29]. However, in the works of Graves-Morris [35] and Aitchison and collaborators [36-38], the possible existence of an additional real, or even complex, contribution was considered within the $K$-matrix formalism. Here, an inhomogeneous real term follows straightforwardly from the reasonable assumption that the produced meson pair originates from an initial $q \bar{q}$ pair.

It is generally agreed that production and scattering have the same singularity structure in the complex energy plane. At first sight, this is not obvious from expressions (15) and (16). However, the second term between braces in Eq. (15) can, using Eq. (11), be rewritten as follows: 


$$
\begin{aligned}
g_{\alpha i} & -2 i \lambda^{2} \sum_{v} \mu_{v} p_{v} j_{\ell}\left(p_{v} r_{0}\right) h_{\ell}^{(1)}\left(p_{v} r_{0}\right) g_{\alpha v} \frac{\mathcal{A}_{i v}^{(\ell)}}{\mathcal{D}^{(\ell)}} \\
& =\frac{1}{\mathcal{D}^{(\ell)}}\left\{g_{\alpha i}+2 i \lambda^{2} \sum_{v} \mu_{v} p_{v} j_{\ell}\left(p_{v} r_{0}\right) h_{\ell}^{(1)}\left(p_{v} r_{0}\right)\left[g_{\alpha i} \mathcal{A}_{v v}^{(\ell)}-g_{\alpha v} \mathcal{A}_{i v}^{(\ell)}\right]\right\} \\
& =\frac{g_{\alpha i}}{\mathcal{D}^{(\ell)}}+2 i \lambda^{2} \sum_{v \neq i} \mu_{v} p_{v} j_{\ell}\left(p_{v} r_{0}\right) h_{\ell}^{(1)}\left(p_{v} r_{0}\right)\left[g_{\alpha i} \frac{\mathcal{A}_{v v}^{(\ell)}}{\mathcal{D}^{(\ell)}}-g_{\alpha v} \frac{\mathcal{A}_{i v}^{(\ell)}}{\mathcal{D}^{(\ell)}}\right] .
\end{aligned}
$$

From this equation it is obvious that, in our approach, scattering and production have exactly the same poles in the complex energy plane, as they share the global denominator $\mathcal{D}$.

\subsection{The central result}

The pole structure of our production amplitude is exhibited very explicitly in formula (18), and shows that it is completely given by $\mathcal{D}$, the very same denominator that determines the pole structure for elastic scattering. The conclusion is that resonance shapes are different for production and scattering because they are largely determined by the respective numerators. Moreover, precisely the numerator $\mathcal{A}_{i i}$ describing elastic scattering in the $i$ th two-meson channel has dropped out of expression (18). Hence, when restricted to a one-channel model, our production amplitude is completely determined by just the denominator $\mathcal{D}$.

The result (18) may be substituted into relation (15). Moreover, using expression (14) for the partial-wave amplitudes, we arrive at

$$
\begin{aligned}
a(\alpha \rightarrow i)= & \frac{\lambda}{\sqrt{\pi}} \sum_{\ell, m}(-i)^{\ell} j_{\ell}\left(p_{i} r_{0}\right) Y_{m}^{(\ell)}\left(\hat{p}_{i}\right) Q_{\ell_{q \bar{q}}}^{(\alpha)}(E) \\
& \times\left\{\frac{g_{\alpha i}}{\mathcal{D}^{(\ell)}}+i \sum_{v \neq i} \mu_{v} p_{v} h_{\ell}^{(1)}\left(p_{v} r_{0}\right)\left[g_{\alpha i} \frac{t_{\ell}(v \rightarrow v)}{j_{\ell}\left(p_{v} r_{0}\right)}-g_{\alpha v} \frac{t_{\ell}(i \rightarrow v)}{j_{\ell}\left(p_{i} r_{0}\right)}\right]\right\} .
\end{aligned}
$$

Eq. (19) is the central result of our paper. It explicitly relates the ingredients of elastic scattering to the amplitude for production in the spectator approximation. We were able to achieve this because in the RSE one can determine in an analytically closed form all terms of the perturbation expansions (5) [2] and (15). Hence, relations (11)-(13) can be derived and explicitly verified. We may thus conclude that at least for a non-relativistic (NR) microscopic model, i.e., at low energies, production and scattering are related to one another through Eq. (19).

\section{4. $P=T / V$}

Expression (18) takes an extremely simple form in the case that all inelasticity is either absent or neglected. For the $\ell$ th partial wave of the production amplitude (15), we then obtain

$$
a^{(\ell)} \propto \lambda_{j_{\ell}}\left(p r_{0}\right) Q_{\ell_{q \bar{q}}}^{(\alpha)}(E) \frac{1}{\mathcal{D}^{(\ell)}}
$$


This is exactly the generic form of the production amplitude used in a paper by Roca et al. [7], when at the $f_{0}(600)$ resonance the inelastic contribution $K K \rightarrow \pi \pi$ is neglected, resulting in $a \propto T / V$. Here, we get in the 1-channel case from Eq. (12) that $\mathcal{A}=Z$, which then precisely yields $T / V=1 / \mathcal{D}$.

\subsection{The meson-loop phase}

In the one-channel approximation, we obtain from the scattering amplitude (5) for the cotangent of the scattering phase shift $\delta^{(\ell)}(E)$ the expression

$$
\operatorname{cotg}\left(\delta^{(\ell)}(E)\right)=\frac{n_{\ell}\left(p r_{0}\right)}{j_{\ell}\left(p r_{0}\right)}-\frac{1}{2 \lambda^{2} \mu p j_{\ell}^{2}\left(p r_{0}\right) \mathcal{A}^{(\ell)}},
$$

where the spherical Neumann function is represented by $n_{\ell}$.

Now, $\mathcal{D}$ in formulae (20) is related to $\mathcal{A}$ in formula (21) through Eq. (11). After some algebra, we get

$$
a^{(\ell)} \propto \lambda_{\ell}\left(p r_{0}\right) Q_{\ell_{q \bar{q}}}^{(\alpha)}(E)\left\{1-\frac{\tan \left(\delta^{(\ell)}(E)\right)}{j_{\ell}\left(p r_{0}\right) / n_{\ell}\left(p r_{0}\right)}\right\} \cos \left(\delta^{(\ell)}(E)\right) \mathrm{e}^{i \delta^{(\ell)}(E)} .
$$

For $S$-waves $(\ell=0)$ this becomes

$$
a^{(0)} \propto \lambda j_{0}\left(p r_{0}\right) Q_{\ell_{\bar{q}}(\alpha)}^{(\alpha)}\left\{1+\frac{\tan \left(\delta^{(0)}(E)\right)}{\tan \left(p r_{0}\right)}\right\} \cos \left(\delta^{(0)}(E)\right) \mathrm{e}^{i \delta^{(0)}(E)}
$$

With respect to the dependence on the phase $\delta^{(0)}(E)$, this expression has exactly the same form as the $S$-wave production amplitude given by Boito and Robilotta [39], which is based on Watson's formalism [6] via the work of Pennington [40]. For the meson-loop phase $\omega(s)$ defined in Ref. [39], we obtain here $p r_{0}$. However, our resonance poles are determined in quite a different manner than in Ref. [39]. Whereas in the RSE the resonance poles are all contained in $\mathcal{A}$ in expression (21) for the cotangent of the phase shift, in the formalism employed in Ref. [39] each of the resonance poles for $S$-wave production has to be put into the corresponding expression by hand, one by one.

\subsection{Breit-Wigner resonances}

Again in the one-channel case, one deduces from Eq. (7) for $\mathcal{D}$ the form

$$
\mathcal{D}^{(\ell)}(E)=1+2 i \lambda^{2}\left\{\sum_{n=0}^{\infty} \frac{\left|F^{(n)}\left(r_{0}\right)\right|^{2}}{E-E_{n}}\right\} \mu p j_{\ell}\left(p r_{0}\right) h_{\ell}^{(1)}\left(p r_{0}\right) .
$$

For small $\lambda$ one finds a zero of $\mathcal{D}$ in the vicinity of $E_{n}$, say at $E_{n}+\Delta E_{n}$, where

$$
\Delta E_{n} \approx 2 \lambda^{2}\left|F^{(n)}\left(r_{0}\right)\right|^{2} \mu_{n} p_{n}\left\{j_{\ell}\left(p_{n} r_{0}\right) n_{\ell}\left(p_{n} r_{0}\right)-i j_{\ell}^{2}\left(p_{n} r_{0}\right)\right\} .
$$

Here, $\mu_{n}$ and $p_{n}$ are the reduced mass and relative linear momentum of the two-meson system at $E=E_{n}$, respectively. Note that the imaginary part of $\Delta E_{n}$ is negative, as it should be for resonance poles in the second Riemann sheet. Below threshold we obtain poles on the real energy axis, since $i j_{\ell} h_{\ell}^{(1)}$ becomes real for purely imaginary arguments. The latter poles 
represent two-meson bound states, as argued above. For the following discussion we shall only consider poles above threshold.

For $\mathcal{D}$ we obtain

$$
\mathcal{D}^{(\ell)}(E) \propto \prod_{n}\left(E-E_{n}-\Delta E_{n}\right) .
$$

Consequently, denoting the residue at the $n$th pole by $\alpha_{n}$, we get

$$
\frac{1}{\mathcal{D}^{(\ell)}(E)} \propto \sum_{n} \frac{\alpha_{n}}{\left(E-E_{n}-\Delta E_{n}\right)},
$$

which is nothing but a Breit-Wigner [41] expansion over a series of resonances, as employed in the isobar formalism [38,42-45].

\subsection{Overlapping resonances}

Of course, things become more involved than in Eq. (27) when $\lambda$ is not small and resonances start to overlap. Overlapping resonances have been studied extensively in the past [46]. Here, it is no longer possible then to deduce simple approximations for expression (24).

Besides extending the formalism of Ref. [6] to coupled channels and overlapping resonances, our work also seems to interpolate between the results of Ref. [40] and Ref. [7].

\subsection{The K-matrix}

The $K$-matrix, which is related to the tangent(s) of the scattering phase shift(s), is defined by

$$
K=T[1+i T]^{-1} .
$$

As follows from the unitarity condition, $K$ is a real (symmetric) matrix for real CM energy E.

In the one-channel approximation and in a particular partial wave, $K$ is given by the inverse of expression (21) for the cotangent of the scattering phase shift. For more channels, relations like Eq. (21) become very complicated expressions in terms of $\mathcal{A}$ and $\mathcal{D}$. The reason is that the inverse of the expression (5) has to be determined. Numerically this is no problem, of course, but analytically it is extremely tedious in the general multichannel case. In particular, for a relation between the common denominator $\mathcal{D}$ and $K$, which is needed for the leading term in expression (19), nothing simple follows. Moreover, the pole positions for both scattering and production stem from $\mathcal{D}$, and not from $K$. Hence, the exercise to express the production amplitude in terms of the $K$-matrix seems pointless.

\section{Summary and concluding remarks}

The two-meson production amplitude (19) has been rigorously calculated, to all orders, from a relatively general expression for a two-meson scattering amplitude (Eq. (5)) dominated by $s$-channel resonances. The latter had already been successfully tested for $c \bar{c}$ and $b \bar{b}$ states, mesons with open charm and bottom, and also in the light-quark sector. 
One might object that a model with no $t$-channel exchanges is too restricted for drawing general conclusions. However, one should be aware of the - quoting Törnqvist [47]"well-known dual-model result for $\bar{q} q$ resonances that a sum of $s$-channel resonances also describes $t$ - and $u$-channel phenomena." In the context of duality, Harari [48] formulated a necessary condition for an $s$-channel description to reproduce certain $t$-channel effects, namely the existence of "strong correlations between the different $s$-channel resonances." Well, this is exactly what our infinite RSE sum over confinement states guarantees. Further proof showing the RSE model to be realistic is its correct threshold behaviour in elastic $\pi \pi$ scattering [49].

Another possible critique of our method could be its NR nature. Nevertheless, in practical phenomenological applications to spectroscopy and elastic scattering, relative momenta and reduced masses in the two-meson channels have been consistently defined in a relativistic way, thus ensuring proper kinematics at much higher energies than the underlying NR formalism seems to support. Such a minimal treatment of relativity is indeed common practice in many relativised quark models. Our successful description of the spectroscopy and scattering properties of the light scalar mesons [50] provides additional evidence that this approach to relativity is reasonable. This is also supported by our very recent first application of the present production formalism in the single-channel case [1].

It thus seems fair to conclude that production amplitudes can in general contain terms which are not proportional to scattering $T$-matrix elements and, moreover, that the proportionality coefficients are complex.

\section{Acknowledgments}

We thank I.J.R. Aitchison, D.V. Bugg, and C. Hanhart for useful discussions. This work was supported in part by the Fundação para a Ciência e a Tecnologia of the Ministério da Ciência, Tecnologia e Ensino Superior of Portugal, under contract PDCT/FP/63907/ 2005.

\section{Appendix A. Generic relation between production and scattering}

In order to arrive at a relation equivalent to Eq. (17) for the here proposed scattering and production amplitudes, we define

$$
T_{i j}^{(\ell)}=-2 \sqrt{\mu_{i} p_{i} \mu_{j} p_{j}} t_{\ell}(i \rightarrow j)=-2 \lambda^{2} \sqrt{\mu_{i} p_{i} \mu_{j} p_{j}} j_{\ell}\left(p_{i} r_{0}\right) j_{\ell}\left(p_{j} r_{0}\right) \frac{\mathcal{A}_{i j}^{(\ell)}}{\mathcal{D}^{(\ell)}} .
$$

For this object, also using relations (6), one easily finds

$$
\begin{aligned}
\sum_{v} T_{i v}^{(\ell) *} T_{v j}^{(\ell)} & =4 \lambda^{4} \sqrt{\mu_{i} p_{i} \mu_{j} p_{j}} j_{\ell}\left(p_{i} r_{0}\right) j_{\ell}\left(p_{j} r_{0}\right) \sum_{v} \mu_{v} p_{v} j_{\ell}^{2}\left(p_{v} r_{0}\right) \frac{\mathcal{A}_{i v}^{(\ell) *} \mathcal{A}_{v j}^{(\ell)}}{\left|\mathcal{D}^{(\ell)}\right|^{2}} \\
& =\frac{\lambda^{2}}{i} \sqrt{\mu_{i} p_{i} \mu_{j} p_{j}} j_{\ell}\left(p_{i} r_{0}\right) j_{\ell}\left(p_{j} r_{0}\right)\left\{\frac{\mathcal{A}_{i j}^{(\ell) *}}{\mathcal{D}^{(\ell) *}}-\frac{\mathcal{A}_{i j}^{(\ell)}}{\mathcal{D}^{(\ell)}}\right\}=\frac{1}{2 i}\left\{T_{i j}^{(\ell)}-T_{i j}^{(\ell) *}\right\} \\
& =\mathfrak{I} m\left(T_{i j}^{(\ell)}\right) .
\end{aligned}
$$


Furthermore, we define

$$
A_{\alpha i}^{(\ell)}=\sqrt{\mu_{i} p_{i}} j_{\ell}\left(p_{i} r_{0}\right)\left\{g_{\alpha i}-2 i \lambda^{2} \sum_{v} \mu_{v} p_{v} j_{\ell}\left(p_{v} r_{0}\right) h_{\ell}^{(1)}\left(p_{v} r_{0}\right) g_{\alpha v} \frac{\mathcal{A}_{i v}^{(\ell)}}{\mathcal{D}^{(\ell)}}\right\},
$$

for which, by substituting definition (A.1), we may also write

$$
A_{\alpha i}^{(\ell)}=g_{\alpha i} j_{\ell}\left(p_{i} r_{0}\right) \sqrt{\mu_{i} p_{i}}+i \sum_{v} g_{\alpha v} \sqrt{\mu_{v} p_{v}} h_{\ell}^{(1)}\left(p_{v} r_{0}\right) T_{i v}^{(\ell)} .
$$

For this object we study, in accordance with relation (17), the imaginary part

$$
\begin{aligned}
& \mathfrak{I} m\left(A_{\alpha i}^{(\ell)}\right)=\sum_{v} g_{\alpha v} \sqrt{\mu_{v} p_{v}} \frac{1}{2 i}\left\{i h_{\ell}^{(1)}\left(p_{v} r_{0}\right) T_{i v}^{(\ell)}+i h_{\ell}^{(2)}\left(p_{v} r_{0}\right) T_{i v}^{(\ell) *}\right\} \\
& =\frac{1}{2} \sum_{v} g_{\alpha v} \sqrt{\mu_{v} p_{v}}\left\{j_{\ell}\left(p_{v} r_{0}\right)\left(T_{i v}^{(\ell)}+T_{i v}^{(\ell) *}\right)+i n_{\ell}\left(p_{v} r_{0}\right)\left(T_{i v}^{(\ell)}-T_{i v}^{(\ell)^{*}}\right)\right\}
\end{aligned}
$$

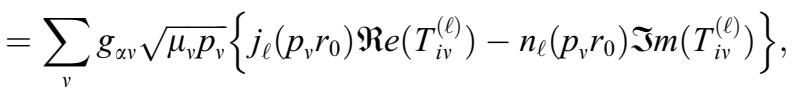

where we denote the spherical Hankel function of the second kind by $h_{\ell}^{(2)}=h_{\ell}^{(1) *}=j_{\ell}-i n_{\ell}$.

Next, we use the fact that $\mathfrak{R} e(T)=T^{*}+i \Im m(T)$, and, moreover, substitute subsequently relations (A.2) and (A.4):

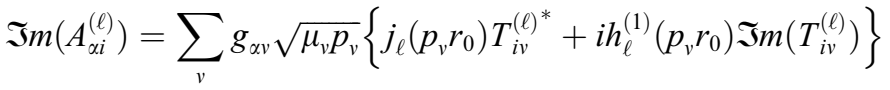

$$
\begin{aligned}
& =\sum_{v} g_{\alpha v} \sqrt{\mu_{v} p_{v}} j_{\ell}\left(p_{v} r_{0}\right) T_{i v}^{(\ell)^{*}}+i \sum_{v^{\prime}} \sum_{v} g_{\alpha v} \sqrt{\mu_{v} p_{v}} h_{\ell}^{(1)}\left(p_{v} r_{0}\right) T_{v^{\prime} v}^{(\ell)} T_{i v^{\prime}}^{(\ell *} \\
& =\sum_{v} T_{i v}^{(\ell)^{*}}\left\{g_{\alpha v} j_{\ell}\left(p_{v} r_{0}\right) \sqrt{\mu_{v} p_{v}}+i \sum_{v^{\prime}} g_{\alpha v^{\prime}} \sqrt{\mu_{v^{\prime}} p_{v^{\prime}}} h_{\ell}^{(1)}\left(p_{v^{\prime}} r_{0}\right) T_{v v^{\prime}}^{(\ell)}\right\} \\
& =\sum_{v} T_{i v}^{(\ell)^{*}} A_{\alpha v}^{(\ell)} .
\end{aligned}
$$

This demonstrates that for our amplitudes a relation exists which is equivalent to the one shown in Eq. (17).

\section{References}

[1] E. van Beveren, G. Rupp, J. Phys. G 34 (2007) 1789. Available from: $<$ hep-ph/0703286 $>$.

[2] E. van Beveren, G. Rupp, Int. J. Theor. Phys. Group Theor. Nonlin. Opt. 11 (2006) 179. Available from: $<$ hep-ph/0304105> .

[3] S. Okubo, Phys. Lett. 5 (1963) 165;

G. Zweig, CERN Reports TH-401 and TH-412.;

See also, D.B. Lichtenberg, S.P. Rosen, Developments in the Quark Theory of Hadrons, vol. 1, 1981, pp. $22-$ 101 ;

J. Iizuka, K. Okada, O. Shito, Prog. Theor. Phys. 35 (1966) 1061.

[4] E. van Beveren, Z. Phys. C 21 (1984) 291. Available from: <hep-ph/0602246>.

[5] W.-M. Yao et al., Particle Data Group Collaboration, J. Phys. G 33 (2006) 1.

[6] K.M. Watson, Phys. Rev. 88 (1952) 1163.

[7] L. Roca, J.E. Palomar, E. Oset, H.C. Chiang, Nucl. Phys. A 744 (2004) 127. Available from: <hep-ph/ 0405228>.

[8] B. Lippmann, J. Schwinger, Phys. Rev. 79 (1950) 469. 
[9] E. van Beveren, C. Dullemond, G. Rupp, Phys. Rev. D 21 (1980) 772 (Erratum-ibid. D 22 (1980) 787).

[10] E. van Beveren, F. Kleefeld, G. Rupp, AIP Conf. Proc. 814 (2006) 143. Available from: $<$ hep-ph/0510120>.

[11] Yu.S. Kalashnikova, Phys. Rev. D 72 (2005) 034010. Available from: $<$ hep-ph/0506270>; T. Barnes, J. Phys. Conf. Ser. 9 (2005) 127. Available from: <hep-ph/0412057>.

[12] V.V. Anisovich, M.A. Matveev, Phys. Atom. Nucl. 67 (2004) 614; Yad. Fiz. 67 (2004) 634. Available from: <hep-ph/0303119>.

[13] J.M. Link et al., FOCUS Collaboration, Phys. Lett. B 585 (2004) 200. Available from: $<$ hep-ex/0312040>.

[14] M.R. Pennington, Prog. Theor. Phys. Suppl. 168 (2007) 143. Available from: <hep-ph/0703256>.

[15] D.V. Bugg, Eur. Phys. J. C 52 (2007) 55. Available from: $<$ hep-ex/0706.1341>.

[16] E. van Beveren, G. Rupp, M.D. Scadron, Phys. Lett. B 495 (2000) 300 (Erratum-ibid. B 509 (2001) 365). Available from: $<$ hep-ph/0009265>.

[17] F. De Fazio, M.R. Pennington, Phys. Lett. B 521 (2001) 15. Available from: <hep-ph/0104289>.

[18] N.N. Achasov, AIP Conf. Proc. 619 (2002) 112. Available from: $<$ hep-ph/0110059>.

[19] T.M. Aliev, A. Özpineci, M. Savc1, Phys. Lett. B 527 (2002) 193. Available from: <hep-ph/0111102>.

[20] C.H. Chen, Phys. Rev. D 67 (2003) 014012. Available from: $<$ hep-ph/0210028>.

[21] P. Colangelo, F. De Fazio, Phys. Lett. B 559 (2003) 49. Available from: <hep-ph/0301267>.

[22] I. Bediaga, M. Nielsen, Phys. Rev. D 68 (2003) 036001. Available from: $<$ hep-ph/0304193>.

[23] V. Baru, J. Haidenbauer, C. Hanhart, Y. Kalashnikova, A. Kudryavtsev, Phys. Lett. B 586 (2004) 53. Available from: $<$ hep-ph/0308129>;

A.V. Anisovich, V.V. Anisovich, V.N. Markov, V.A. Nikonov, A.V. Sarantsev, Phys. Atom. Nucl. 68 (2005) 1554;

Yad. Fiz. 68 (2005) 1614. Available from: $<$ hep-ph/0403123>;

Yu.S. Kalashnikova, A.E. Kudryavtsev, A.V. Nefediev, C. Hanhart, J. Haidenbauer, Eur. Phys. J. A 24 (2005) 437. Available from: <hep-ph/0412340>.

[24] D.V. Bugg, Eur. Phys. J. C 47 (2006) 45. Available from: <hep-ex/0603023>.

[25] V.V. Anisovich. Available from: $<$ hep-ph/0606266 $>$.

[26] A.K. Giri, B. Mawlong, R. Mohanta, Phys. Rev. D 74 (2006) 114001. Available from: $<$ hep-ph/0608088>.

[27] S.U. Chung, J. Brose, R. Hackmann, E. Klempt, S. Spanier, C. Strassburger, Ann. Phys. 507 (1995) 404.

[28] K.L. Au, D. Morgan, M.R. Pennington, Phys. Rev. D 35 (1987) 1633.

[29] M. Boglione, M.R. Pennington, Eur. Phys. J. C 30 (2003) 503. Available from: $<$ hep-ph/0303200>.

[30] P. Dini, FOCUS Collaboration, Int. J. Mod. Phys. A 20 (2005) 482.

[31] J.M. Link et al., FOCUS Collaboration, M.R. Pennington, Phys. Lett. B 653 (2007) 1. Available from: $<$ hep$\mathrm{ex} / 0705.2248>$.

[32] M. Ishida, S. Ishida, T. Ishida, Prog. Theor. Phys. 99 (1998) 1031. Available from: <hep-ph/9805319>; M. Ishida, S. Ishida and T. Ishida. Available from: $<$ hep-ph/9802272>.

[33] M. Ishida, AIP Conf. Proc. 688 (2004) 18.

[34] U.G. Meißner, J.A. Oller, Nucl. Phys. A 679 (2001) 671. Available from: $<$ hep-ph/0005253 $>$.

[35] P.R. Graves-Morris, Nuovo Cim. A 50 (1967) 681.

[36] M.G. Bowler, M.A.V. Game, I.J.R. Aitchison, J.B. Dainton, Nucl. Phys. B 97 (1975) 227.

[37] I.J.R. Aitchison, M.G. Bowler, J. Phys. G 3 (1977) 1503.

[38] I.J.R. Aitchison, Phys. Lett. B 84 (1979) 349.

[39] D.R. Boito, M.R. Robilotta, Phys. Rev. D 76 (2007) 094011. Available from: <hep-ph/0705.3260>.

[40] M.R. Pennington. Available from: $<$ hep-ph/9710456>.

[41] G. Breit, E. Wigner, Phys. Rev. 49 (1936) 519.

[42] D. Herndon, P. Soding, R.J. Cashmore, Phys. Rev. D 11 (1975) 3165.

[43] D. Herndon, R. Longacre, L.R. Miller, A.H. Rosenfeld, G. Smadja, P. Soding, R.J. Cashmore, D.W.G.S. Leith, Phys. Rev. D 11 (1975) 3183.

[44] V.V. Anisovich, D.V. Bugg, A.V. Sarantsev, B.S. Zou, Phys. Rev. D 50 (1994) 1972.

[45] C.L. Korpa, M.F.M. Lutz, Nucl. Phys. A 742 (2004) 305. Available from: $<$ nucl-th/0306063>.

[46] I.J.R. Aitchison, Nucl. Phys. A 189 (1972) 417.

[47] N.A. Tornqvist, M. Roos, Phys. Rev. Lett. 77 (1996) 2333. Available from: <hep-ph/9610527>.

[48] H. Harari, Phys. Rev. Lett. 26 (1971) 1400.

[49] E. van Beveren and G. Rupp. Available from: $<$ hep-ph/0702117 $>$.

[50] E. van Beveren, D.V. Bugg, F. Kleefeld, G. Rupp, Phys. Lett. B 641 (2006) 265. Available from: <hep-ph/ 0606022>. 